\title{
Quelle progression des compétences interprétatives des élèves de 12 à 15 ans et quelle prise en compte par leurs enseignants?
}

Une étude internationale

What progression of pupils' interpretative skills from 12 to 15 years old and what is taken into account by their teachers? An international study

Jean-Louis Dufays, Magali Brunel, Judith Émery-Bruneau, Marie Barthélemy, Vincent Capt, Isabelle Brun-Lacour, Sonya Florey et Martin Lépine

\section{(2) OpenEdition}

\section{Journals}

Édition électronique

URL : https://journals.openedition.org/pratiques/9007

DOI : $10.4000 /$ pratiques.9007

ISSN : 2425-2042

Éditeur

Centre de recherche sur les médiations (CREM)

Référence électronique

Jean-Louis Dufays, Magali Brunel, Judith Émery-Bruneau, Marie Barthélemy, Vincent Capt, Isabelle Brun-Lacour, Sonya Florey et Martin Lépine, «Quelle progression des compétences interprétatives des élèves de 12 à 15 ans et quelle prise en compte par leurs enseignants ? », Pratiques [En ligne], 187-188 | 2020, mis en ligne le 12 décembre 2020, consulté le 21 juillet 2021. URL : http:// journals.openedition.org/pratiques/9007 ; DOI : https://doi.org/10.4000/pratiques.9007 


\section{Quelle progression des compétences interprétatives des élèves de 12 à 15 ans et quelle prise en compte par leurs enseignants?}

Une étude internationale

What progression of pupils' interpretative skills from 12 to 15 years old and what is taken into account by their teachers? An international study

Jean-Louis Dufays, Magali Brunel, Judith Émery-Bruneau, Marie Barthélemy, Vincent Capt, Isabelle Brun-Lacour, Sonya Florey et Martin Lépine

\section{Introduction}

\section{Le « projet Gary » en deux mots}

1 Le projet dans lequel s'inscrit la présente contribution est né en 2014 et rassemble aujourd'hui neuf chercheurs issus de quatre pays francophones (Belgique, France, Suisse et Québec). Fondé sur un large corpus dont la récolte devrait s'achever en 2021, il s'est donné comme objectif d'étudier dans les quatre pays précités et cela à trois niveaux du curriculum de l'école obligatoire - à savoir les niveaux 4, 7 et 10, qui correspondent aux élèves âgés de 9-10 ans, 12-13 ans et 15-16 ans - les trois questions de recherche suivantes:

- Comment se caractérisent et évoluent les compétences de lecture de textes littéraires des élèves?

- Comment se caractérisent et évoluent les pratiques enseignantes centrées sur ces compétences?

-Quelles relations peut-on établir, si cela est possible, entre ces deux ensembles de données? 
2 L'idée qui motive le projet est qu'une meilleure connaissance des réalités de l'apprentissage et de l'enseignement de la lecture ainsi que de leurs liens réciproques permettrait de mieux informer et former les enseignants et, partant, les aiderait à contrer les effets néfastes des ruptures qui affectent la progression tant des pratiques d'enseignement que des effets d'apprentissage (Dufays, 2010 ; Dumortier, Van Beveren \& Vrydaghs, 2012 ; Brunel, Dufays \& Émery-Bruneau, 2017) en vue de mieux garantir la réussite et la progression de leurs élèves.

3 Aux trois questions générales qui fondent la recherche sont venues se greffer au fil du temps différentes questions connexes, comme celle de la place accordée à l'altérité comprise comme l'ouverture éthique aux différences - dans les productions des élèves et les pratiques enseignantes (Brunel et al., à paraitre), celle du traitement de l'appréciation (Brunel et al., 2018) ou encore celle du regard des enseignants sur l'expérience de cette recherche (Brun-Lacour \& Dufays, à paraitre). Dans la présente contribution nous nous intéressons à la manière dont les élèves et les enseignants de deux des trois niveaux scolaires concernés (les niveaux 7 et 10) gèrent les activités interprétatives.

\section{Cadre méthodologique}

4 La récolte des données, qui, au terme du projet, devrait concerner 96 classes (24 par pays et 32 par niveau) et environ 2500 élèves, est réalisée dans des classes d'indice socio-économique moyen, ce qui permet de neutraliser cette variable.

5 Cette récolte s'appuie sur un texte-support unique, à savoir la nouvelle de R. Gary « J'ai soif d'innocence ${ }^{1}$ ", qui a été choisie à la fois pour sa lisibilité - certes relative - par les élèves des différents niveaux scolaires ciblés, pour l'intérêt supposé des valeurs que ce récit met en scène ainsi que pour la diversité présumée de ses significations. La trame est la suivante: le narrateur, dégouté du mercantilisme de notre société moderne, quitte son pays en quête d'un mode de vie plus altruiste. Après un passage décevant à Tahiti, il arrive sur Taratora, une ile des Marquises, où il croit trouver une société idéale auprès d'une population qui se met à son service, ainsi qu'une véritable amitié avec Taratonga, femme généreuse et soucieuse du bien-être de tous les habitants. Mais voilà que Taratonga lui offre des gâteaux enveloppés dans ce qu'il croit être des toiles de Gauguin. Celles-ci réveillent ses intérêts et, sans rien dire aux habitants ni à Taratonga - à qui il cède cependant sa montre et ses économies-, le narrateur s'apprête à ramener les toiles dans son pays, imaginant le profit qu'il pourrait tirer d'une telle découverte. Sur le chemin du retour, il est détrompé par un hôtelier qui lui révèle que Taratonga imite fort bien des œuvres de Gauguin et les revend à l'étranger. Ses rêves de richesse s'envolent ainsi que ses illusions sur le monde idéal de l'ile, sur les valeurs de l'innocence et de la pureté.

6 Le protocole de récolte des données comporte deux temps correspondant à deux séances de cours, dont la durée oscille le plus souvent entre 40 et 50 minutes. Au cours de la première séance, les élèves lisent le texte sans intervention de l'enseignant et répondent en autonomie à un questionnaire constitué de trois questions : 1) Présente ce récit en détail, comme si tu t'adressais à quelqu'un qui ne le connait pas ; 2) Ce texte t'a-t-il plu? Pourquoi ? 3) Selon toi, que cherche à nous dire l'auteur ? Au cours de la seconde séance, qui est filmée puis transcrite par un membre de l'équipe de recherche, les enseignants exploitent le texte librement, selon leur habitude. Ainsi disposons-nous 
de deux ensembles de données : les réponses des élèves au questionnaire et les films et verbatims des séances de classe.

7 La présente étude concerne les données récoltées dans 16 classes $^{2}$ des niveaux 7 et 10 (8 classes de chaque niveau, 2 par pays par niveau), ce qui représente un huitième des données visées, et correspond à 377 réponses au questionnaire, réparties comme suit :

Tableau $1:$ Nombre de questionnaires recueillis dans les quatre pays

\begin{tabular}{|l|l|l|l|l|l|}
\hline & Belgique & France & Québec & Suisse & Total \\
\hline Élèves du niveau 7 (12 ans) & 44 & 52 & 58 & 38 & 192 \\
\hline Élèves du niveau 10 (15 ans) & 50 & 54 & 49 & 32 & 185 \\
\hline Total & 94 & 106 & 107 & 70 & 377 \\
\hline
\end{tabular}

Il va de soi qu'un tel échantillon, qui plus est de convenance, est encore trop limité pour autoriser quelque généralisation que ce soit. Les analyses qui vont suivre ont donc une visée exploratoire : leur but est à la fois d'éprouver la solidité des outils d'analyse mobilisés et d'identifier des tendances en vue de formuler de premières hypothèses qu'il s'agira de valider, d'infirmer ou d'affiner dans la suite de la recherche.

\section{Cadre conceptuel}

Puisque l'étude porte à la fois sur les lectures des élèves et sur les pratiques de leurs enseignants, elle se fonde sur un double cadre théorique.

Le premier concerne la définition et l'analyse des processus de lecture qui ont été développées tant par des théoriciens de la littérature comme H. R. Jauss, W. Iser, U. Eco, M. Picard, V. Jouve ou Y. Citton que par des didacticiens comme A. Rouxel, C. Tauveron, J.-L. Dufays, S. Ahr ou B. Louichon. Ce champ ayant déjà fait l'objet de nombreuses synthèses, nous ne nous y attarderons pas ici, sinon pour préciser la conception de l'interprétation qui servira de base à l'analyse. En nous appuyant sur les auteurs précités, nous définissons l'interprétation comme l'explicitation d'éléments non-dits, susceptibles de lecture plurielle (polysémie), et qui peuvent concerner un élément du texte (action, construction narratologique, personnage) ou le texte dans son ensemble (la « leçon » du récit). Ainsi l'interprétation conçue peut-elle se fonder sur des données variées qui peuvent être internes ou externes au texte, intersubjectives (c'est-à-dire linguistiques, stylistiques, ou relatives à un contexte historique, sociologique, littéraire, intertextuel, cotextuel, biographique, psychanalytique...) ou subjectives (c'est-à-dire propres au lecteur et relever de ses souvenirs ou de ses associations libres), et dirigées vers l'amont du texte (c'est-à-dire sa contextualisation) ou vers son aval (c'est-à-dire son actualisation).

Plus précisément, dans leur étude relative aux gestes professionnels des enseignants qui travaillent l'interprétation avec leurs élèves, A. Jorro et H. Crocé-Spinelli (2010) soulignent que l'interprétation ne sert pas à reformuler "un sens caché » mais à " interpréter le texte au sens de P. Ricœur [1990], dans une dialectique compréhensionexplication (arc herméneutique)» et à construire des sens, des significations (voire vivre des sensations, ressentir...) à l'aide des indices du texte - en se référant alors aux "droits du texte», qui servent de garde-fou aux surinterprétations et aux 
interprétations «délirantes ». Nous nous référons par ailleurs à la typologie qu'a établie M. Sauvaire (2013) des différents types de ressources sur lesquelles les élèves se fondent pour formuler leurs interprétations en tant que « sujets lecteurs ", à savoir les ressources cognitives, épistémiques, psychoaffectives, axiologiques et éthiques et socioculturelles ${ }^{3}$.

12 À côté de la compréhension et de l'interprétation, nous nous intéressons dans notre recherche à deux autres opérations de lecture ou activités liées à la lecture: l'appréciation, dont nous avons tracé les contours dans une précédente étude (Brunel \& al., 2018) $)^{4}$, et les apports culturels, qui consistent à convoquer des savoirs en appui à la lecture, lesquels savoirs peuvent constituer une fin en soi ou bien être mis au service d'une des trois opérations précitées.

13 Le second cadre conceptuel qui fonde notre recherche est celui de l'analyse des pratiques enseignantes. Nous privilégions désormais ${ }^{5}$ trois cadres d'analyse qui nous paraissent bien rendre compte de la spécificité de nos données, à savoir :

- la typologie des schèmes didactiques, qui a été mise au point dans une étude de $\mathrm{S}$. Marlair \& J.L. Dufays (2008) et nous a amenés à distinguer six schèmes transversaux : l'exposé magistral, le balisage méthodologique, le cours dialogué, le travail de groupe, le travail individuel et les intermèdes ;

- la distinction que B. Schneuwly \& J. Dolz (2009) ont opérée entre quatre gestes didactiques fondamentaux : la mobilisation de la mémoire, la mise en œuvre de dispositifs (qui inclut les gestes de présentification et d'élémentarisation de l'objet enseigné), les gestes de régulation et d'évaluation des apprentissages et les gestes d'institutionnalisation des contenus enseignés ;

- et la typologie que S. Aeby Daghé (2014) a établie entre douze " genres d'activité scolaire » ou "GAS", dont nous rappelons la liste ci-dessous, en renvoyant à cette auteure pour la définition précise de ces différentes catégories :

Tableau 2 : Les genres d'activité scolaire relatifs à la lecture des textes littéraires selon S. Aeby Daghé (2014)

\begin{tabular}{|l|l|}
\hline \multirow{4}{*}{ Appropriation } & 1- Résumé \\
\cline { 2 - 2 } & 2- Lecture à haute voix \\
\cline { 2 - 2 } & 3- Lecture maison \\
\hline \multirow{5}{*}{ Dommentaire du texte } & 4- Explication de texte \\
\cline { 2 - 3 } & 5- Compréhension \\
\cline { 2 - 3 } & 6- Étude de dimensions de l'objet \\
\hline \multirow{5}{*}{ Discours sur la production } & 7- Mise en réseaux \\
\cline { 2 - 3 } & 8- Présentation de texte \\
\cline { 2 - 3 } & 9- Débat interprétatif \\
\hline & 10- Discussion thématique \\
\cline { 2 - 3 } & 11- Expression d'avis sur le texte \\
\cline { 2 - 3 } & 12- Production de texte \\
\hline
\end{tabular}


Opérations, schèmes, gestes et GAS : ce sont ces quatre approches qui seront mobilisées dans le présent article pour analyser les pratiques enseignantes.

L'étude qui suit se divise donc en trois volets : nous nous intéresserons tour à tour aux compétences des élèves, aux pratiques de leurs enseignants et, si cela est possible, aux relations repérables entre les unes et les autres.

\section{Du côté des élèves : quelles compétences interprétatives aux niveaux 7 et 10 ?}

Comme nous l'avons mentionné, notre questionnaire comportait trois questions à développement, qui visaient à cerner 1) la compréhension; 2) l'appréciation ; 3) l'interprétation. Dans la présente contribution, nous présentons les résultats relatifs à la dernière question, qui invitait les élèves à faire ressortir leurs interprétations de la nouvelle de R.Gary en leur demandant: "Selon toi, que cherche à nous dire l'auteur?».

Les réponses relatives à l'interprétation ont été analysées à l'aide d'une grille comportant trois grandes sections (voir tableau 3). Tout d'abord, nous avons listé les réponses vides, les interprétations délirantes, les contresens ou autres énoncés qui ne proposaient pas une interprétation de la nouvelle de R. Gary. Ensuite, nous avons listé les types d'interprétation qui émergeaient au moins une fois dans les réponses des élèves, ce qui nous a permis d'identifier six interprétations distinctes. Puis, nous avons cherché à dresser une typologie des éventuelles justifications convoquées par les élèves pour appuyer leurs interprétations. À l'instar de A. Jorro et H. Crocé-Spinelli (2010), nous avons porté attention non seulement aux interprétations générales formulées par les élèves, mais aussi à leurs justifications, que nous avons analysées à la lumière de la typologie de M. Sauvaire (2013), ce qui nous a permis d'y distinguer des ressources cognitives, épistémiques, psychoaffectives, axiologiques et éthiques.

Tableau 3 : exemple de la grille utilisée pour analyser la $3^{e}$ question posée aux élèves (interprétation)

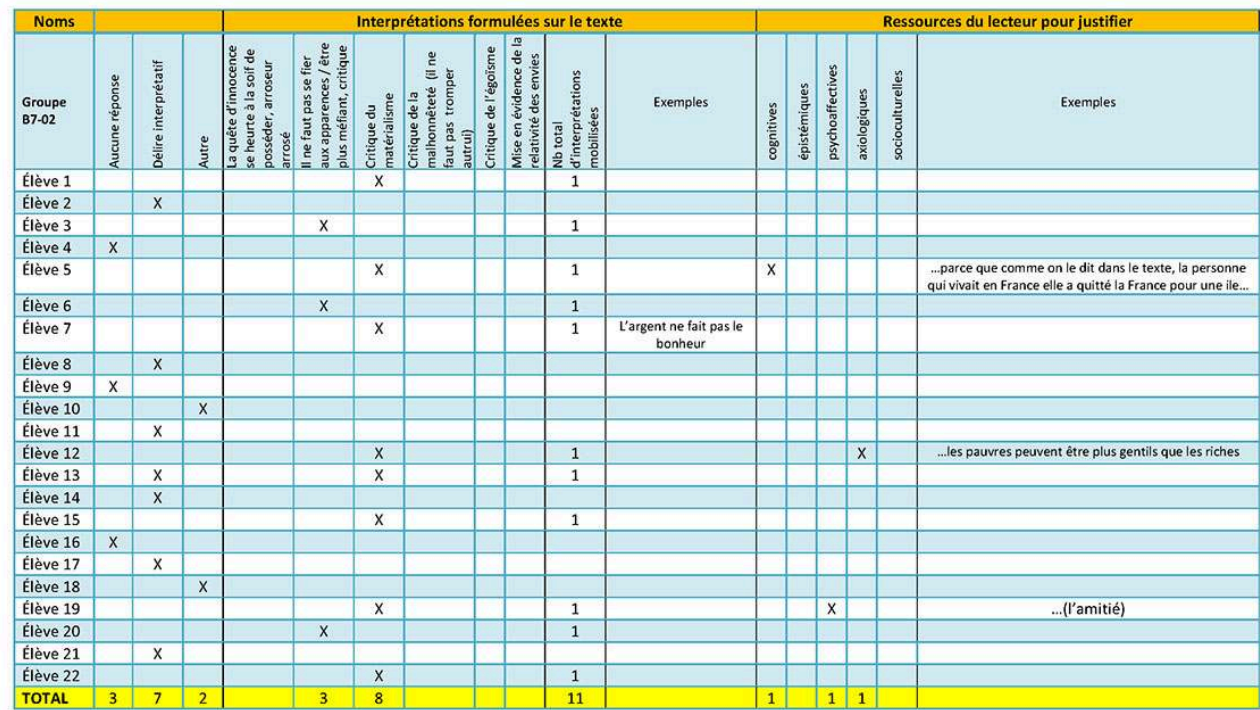




\subsection{Résultats des élèves du niveau 7}

Le tableau suivant présente la somme des résultats obtenus dans notre corpus de questionnaires remplis par les élèves du niveau 7. Les résultats quantifiés ont aussi été traduits en pourcentage afin de faciliter les comparaisons entre les niveaux et les pays.

Tableau 4 : synthèse des résultats « interprétation » des élèves du niveau $7^{6}$

\begin{tabular}{|c|c|c|c|c|c|c|c|c|c|c|c|c|c|c|c|}
\hline \multirow[t]{2}{*}{ Pays } & & & & \multicolumn{6}{|c|}{ Interprétations formulées sur le texte } & \multicolumn{6}{|c|}{$\begin{array}{l}\text { Ressources du lecteur } \\
\text { pour justifier }\end{array}$} \\
\hline & 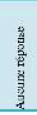 & 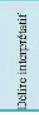 & $\frac{2}{2}$ & 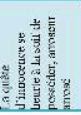 & 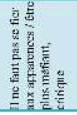 & 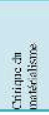 & 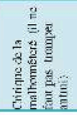 & 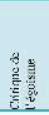 & 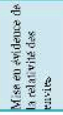 & 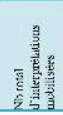 & $\begin{array}{l}\frac{b}{3} \\
\frac{1}{2} \\
\frac{1}{2}\end{array}$ & 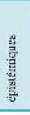 & $\begin{array}{l}\text { है } \\
\text { है } \\
\text { है } \\
\end{array}$ & 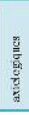 & $\begin{array}{l}\frac{2}{2} \\
\frac{2}{2} \\
\frac{2}{2} \\
\frac{2}{2} \\
\end{array}$ \\
\hline \multirow{2}{*}{ 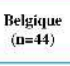 } & 6 & 1.3 & 4 & 0 & 8 & 15 & 1 & 1 & 0 & -- & 2 & 0 & 1 & 2 & 0 \\
\hline & \multicolumn{3}{|c|}{$23(52,3 \%)$} & \multicolumn{6}{|c|}{$25(56,8 \%)$} & $\ldots$ & \multicolumn{5}{|c|}{$5(11,4 \%)$} \\
\hline \multirow{2}{*}{$\begin{array}{l}\text { France } \\
(\mathbf{n}=52)\end{array}$} & 4 & 4 & 6 & 3 & 11 & 24 & 8 & 5 & 4 & 1,27 & 0 & 0 & 0 & 0 & 0 \\
\hline & \multicolumn{3}{|c|}{$14(26,9 \%)$} & \multicolumn{6}{|c|}{$55(105,8 \%)$} & -- & \multicolumn{5}{|c|}{$0(0 \%)$} \\
\hline \multirow{2}{*}{$\begin{array}{c}\text { Quélece } \\
(\mathbf{n}=\Sigma \$ \text { ) }\end{array}$} & 2 & 19 & 5 & 3 & 7 & 22 & 2 & 1 & 0 & -- & 0 & 0 & 0 & 0 & 0 \\
\hline & \multicolumn{3}{|c|}{$26(44,8 \%)$} & \multicolumn{6}{|c|}{$35(60,3 \%)$} &.- & \multicolumn{5}{|c|}{$\mathbf{0}(0 \%)$} \\
\hline \multirow{2}{*}{$\begin{array}{l}\text { Sulkse } \\
(\mathbf{n}=3 \mathbf{3 s})\end{array}$} & 1 & 2 & 0 & 1 & 15 & 15 & 2 & 2 & 5 & 1,23 & 0 & 0 & 0 & 0 & 0 \\
\hline & \multicolumn{3}{|c|}{$3(7,9 \%)$} & \multicolumn{6}{|c|}{$40(105.3 \%)$} & -- & \multicolumn{5}{|c|}{$0(0 \%)$} \\
\hline \multirow{2}{*}{$\begin{array}{l}\text { TOTAL } \\
(\mathrm{n}=192)\end{array}$} & 1.3 & 38 & 15 & 7 & 41 & 76 & 13 & 9 & 9 & + & 2 & 0 & 1 & 2 & 10 \\
\hline & \multicolumn{3}{|c|}{$66(34,4 \%)$} & \multicolumn{6}{|c|}{$157(81,8 \%)$} & ... & \multicolumn{5}{|c|}{$s(2,6 \%)$} \\
\hline
\end{tabular}

19 D'entrée de jeu, nous observons dans la seconde colonne qu'un tiers des élèves ( $34,4 \%)$ n'ont pas été en mesure de formuler des interprétations sur le texte. Dans la partie "interprétations formulées sur le texte», le pourcentage indiqué correspond au nombre d'interprétations rapporté au nombre d'élèves, sachant que certains d'entre eux proposent plusieurs interprétations. Cela explique qu'un peu plus de $80 \%$ des copies présentaient des interprétations, qui relevaient principalement soit d'une critique du matérialisme, du type «l'argent ne fait pas le bonheur ", souvent formulée dans des énoncés figés sous forme de proverbes, soit d'appels à la vigilance, sur le mode « il ne faut pas se fier à autrui » ou "il faut être plus critique et méfiant des autres ». Ces résultats pourraient s'expliquer de diverses manières : par le texte lui-même, qui conduit à dégager ces deux «leçons» de vie; par la société néolibérale dans laquelle nous vivons, qui fait que les élèves sont constamment confrontés à l'idée que l'argent mène le monde; et par l'empathie des jeunes lecteurs pour les relations d'amitié, les actions et les motivations des personnages, qui a déjà été signalée par plusieurs chercheurs (Hébert, 2013 ; Larrivé, 2015 ; Thompson, 1987)... Mais il faut aussi envisager l'impact possible d'un certain habitus scolaire qui aurait privilégié ce type d'interprétation. Enfin, moins de $3 \%$ des copies contiennent une justification, même minimale, pour appuyer leurs interprétations. Il semble donc que, chez les élèves du niveau 7, la justification n'est pas acquise ou ne va pas de soi dans leur discours de lecteurs lorsqu'elle n'est pas directement sollicitée au sein de la question posée.

\subsection{Résultats des élèves du niveau 10}

Le tableau suivant présente la somme des résultats obtenus dans notre corpus de questionnaires remplis par les élèves du niveau 10. 
Tableau 5 : synthèse des résultats « interprétation » des élèves du niveau 10

\begin{tabular}{|c|c|c|c|c|c|c|c|c|c|c|c|c|c|c|c|}
\hline \multirow[t]{2}{*}{ Pays } & & & & \multicolumn{6}{|c|}{ Interprétations formulées sur le texte } & & \multicolumn{5}{|c|}{$\begin{array}{l}\text { Ressources du lecteur } \\
\text { pour justifier }\end{array}$} \\
\hline & 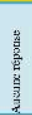 & 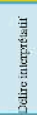 & $\frac{y}{2}$ & 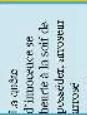 & 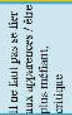 & 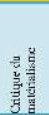 & 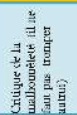 & 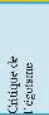 & 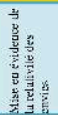 & 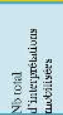 & $\frac{3}{3}$ & 竞 & $\begin{array}{l}0 \\
\frac{1}{2} \\
\frac{2}{2} \\
\frac{2}{2} \\
\end{array}$ & 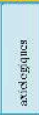 & $\begin{array}{l}\frac{y}{2} \\
\frac{2}{2} \\
\frac{2}{2} \\
\end{array}$ \\
\hline \multirow{2}{*}{$\begin{array}{c}\text { Belgique } \\
(\mathbf{n}=50)\end{array}$} & 2 & 11 & 6 & 8 & 14 & 21 & 2 & 3 & 0 & -.. & 5 & 0 & 2 & 0 & 3 \\
\hline & \multicolumn{3}{|c|}{$19(38 \%)$} & \multicolumn{6}{|c|}{$48(96 \%)$} & $\ldots$ & \multicolumn{5}{|c|}{$10(20 \%)$} \\
\hline \multirow{2}{*}{$\begin{array}{l}\text { France } \\
(\mathrm{u}=54)\end{array}$} & 8 & 9 & 1 & 5 & 11 & 17 & 0 & 3 & 14 & & 10 & 0 & 0 & 0 & 4 \\
\hline & \multicolumn{3}{|c|}{$18(33,3 \%)$} & \multicolumn{6}{|c|}{$50(92,6 \%)$} & -- & \multicolumn{5}{|c|}{$14(25,9 \%)$} \\
\hline \multirow{2}{*}{$\begin{array}{l}\text { Quêber } \\
\text { (n=49) }\end{array}$} & 0 & 7 & 5 & 7 & 14 & 17 & 1 & 1 & 0 & $\ldots$ & 1 & 0 & 3 & 3 & 1 \\
\hline & \multicolumn{3}{|c|}{$12(24,5 \%)$} & \multicolumn{6}{|c|}{$40(81,6 \%)$} & ... & \multicolumn{5}{|c|}{$8(16,3 \%)$} \\
\hline \multirow{2}{*}{$\begin{array}{c}\text { Sulsse } \\
(\mathrm{D}=32)\end{array}$} & 0 & 2 & 0 & 7 & 5 & 13 & 0 & 3 & 19 & 1,23 & 4 & 0 & 0 & 2 & 2 \\
\hline & \multicolumn{3}{|c|}{$2(6,3 \%)$} & \multicolumn{6}{|c|}{$37(115,6 \%)$} & $\ldots$ & \multicolumn{5}{|c|}{$8(25 \%)$} \\
\hline \multirow{2}{*}{$\begin{array}{l}\text { TOTAL } \\
(\mathrm{n}=185)\end{array}$} & 10 & 29 & 12 & 27 & 44 & 68 & 3 & 10 & 33 & +-- & 20 & 0 & 5 & 5 & 10 \\
\hline & \multicolumn{3}{|c|}{$51(27,6 \%)$} & \multicolumn{6}{|c|}{$185(100 \%)$} & -- & \multicolumn{5}{|c|}{$40(21,6 \%)$} \\
\hline
\end{tabular}

${ }_{21} \mathrm{Si}$, au niveau 7, un tiers des élèves glissaient vers le délire interprétatif ou ne répondaient pas, seulement un quart des élèves $(27,6 \%)$ du niveau 10 ont fait de même, ce qui est un premier signe de progression entre les deux niveaux. Autre signe de progression, les interprétations sont beaucoup plus diversifiées chez les élèves du niveau 10, ce qui indique que leur réception se densifie et se complexifie. Les deux interprétations qui arrivent en tête sont néanmoins les mêmes que celles des élèves du niveau 7 (critique du matérialisme, « il ne faut pas se fier aux apparences »), auxquelles s'ajoutent la mise en évidence de la relativité des envies et l'idée « d'arroseur arrosé ». Enfin, nous voyons un indice fort des compétences des élèves du niveau 10 à interpréter dans leur capacité à expliquer/justifier ce qu'ils avancent, car $21 \%$ des copies contiennent au moins une justification, ce qui représente selon nous un indice de leur habitus scolaire (selon lequel justifier consiste à donner des « preuves»).

\subsection{Quelles progressions entre les niveaux ?}

On le voit, les compétences des élèves en interprétation progressent de façon manifeste entre le niveau 7 et le niveau 10 sur divers plans. En particulier, le nombre de réponses incomplètes, de contresens et de délires interprétatifs passe de $34,4 \%$ à 27,6\%. Deux hypothèses permettent d'expliquer, du moins en partie, ces réponses problématiques. D'abord, au regard du temps dont les élèves ont disposé pour lire le texte (20 à 30 minutes, quel que soit le niveau ; et sans avoir consulté préalablement le questionnaire pour se donner une intention de lecture précise), puis pour répondre aux trois questions du questionnaire (à nouveau 20 à 30 minutes), comme la question d'interprétation était la dernière, peut-être plusieurs élèves ont-ils manqué de temps pour réaliser la tâche. En second lieu, ce résultat pourrait aussi s'expliquer par les difficultés que présente ce texte pour certains élèves, particulièrement en ce qui concerne ses référents culturels, son vocabulaire, sa longueur et son genre, avec lequel les plus jeunes sont moins familiers, comme ils l'ont indiqué dans leurs réponses à la question d'appréciation (voir notre étude dans Brunel et al., 2018). Cependant, cette hypothèse doit être nuancée dans la mesure où le texte est publié dans un recueil de nouvelles destiné aux élèves de 13-14 ans ${ }^{7}$.

Les interprétations sont en outre plus diversifiées au niveau 10, de même que les justifications, qui augmentent significativement, de $2 \%$ à $21 \%$. Pour expliquer ce résultat, nous avançons l'hypothèse que les élèves du niveau 10 ont forcément vécu plus d'expériences de lecture, acquis plus de connaissances et ont davantage de 
référents culturels que les plus jeunes, mais ils auraient aussi développé plus de réflexions sur le monde - et pas seulement des préoccupations tournées vers la morale de l'histoire ou vers l'empathie envers les personnages comme les plus jeunes. La compétence à interpréter progresse donc au fil du secondaire, pour une majorité d'élèves. Il est possible aussi que cette progression soit due à l'habitus scolaire, dans la mesure où, dans les différents pays concernés par notre étude, c'est surtout au niveau 10 que les élèves apprennent à argumenter et à justifier en étudiant le texte argumenté: nous ne serions pas étonnés qu'ils aient transféré ces apprentissages ${ }^{8}$. Enfin, l'hypothèse de "l'effet enseignement" pourrait aussi être avancée, dans la mesure où, souvent, au fil de la scolarité, les enseignants demandent aux élèves de "prouver» ce qu'ils avancent, ce qui d'ailleurs relève aussi de l'apprentissage de l'argumentation; ainsi, à 15-16 ans, ils sont plus préparés à donner des réponses scolaires à un questionnaire scolaire. Il s'agit là d'une conséquence de la disciplination, aussi observée par C. Ronveaux et B. Schneuwly (2018). En somme l'analyse générale des réponses des élèves au volet "interprétation " permet de conclure qu'il y a bien une certaine progression entre les niveaux 7 et 10, en particulier pour ce qui concerne la richesse des interprétations et la présence ou non d'une justification à celles-ci.

\section{Du côté des pratiques d'enseignement: quelle place pour l'interprétation?}

\subsection{Choix méthodologiques}

Après avoir ainsi décrit l'évolution des performances des élèves, nous en venons au deuxième volet de notre étude, celui de l'analyse des pratiques mises en œuvre par les 14 professeurs des 16 classes du présent corpus pour étudier avec leurs élèves le texte de R. Gary. Cette analyse s'appuie d'une part sur les quatre typologies que nous avons évoquées dans notre introduction, à savoir celles des opérations de lecture, des schèmes didactiques transversaux, des gestes didactiques fondamentaux et des genres d'activités scolaires, et d'autre part sur la place que le travail interprétatif réserve aux différents aspects du texte (titre, personnages, structure narrative...). Notre analyse envisage donc cinq focales :

1. Quelle part le travail interprétatif occupe-t-il dans les séances par rapport aux activités qui portent sur la compréhension et sur l'appréciation?

2. Quels sont les aspects du texte qui font l'objet de l'activité interprétative ?

3. Quels schèmes d'action les enseignants déploient-ils pour solliciter des interprétations ?

4. Quels gestes didactiques privilégient-ils pour ce faire?

5. Et à quels genres d'activité scolaires ou « GAS » font-ils appel ?

Pour analyser les verbatims des 14 séances que nous avons transcrits, nous avons recouru à cinq grilles d'analyse ${ }^{9}$ et avons calculé, pour chacune, la part de leurs différentes catégories au sein des leçons.

Le tableau ci-dessous ${ }^{10}$ présente l'ensemble des données ainsi codées pour les focales " opérations ", « schèmes » et « gestes » : 
Tableau 6. Codage des séances en termes d'opérations de lecture, de schèmes transversaux et de gestes didactiques

\begin{tabular}{|c|c|c|c|c|c|c|c|c|c|c|c|c|c|c|c|c|}
\hline \multirow[b]{2}{*}{ Identifiant } & \multicolumn{5}{|c|}{ OPÉRATIONS DE LECTURE } & \multicolumn{6}{|c|}{ SCHEMES TRANSVERSAUX } & \multicolumn{5}{|c|}{ GESTES DIDACTIQUES FONDAMENTAUX } \\
\hline & $\begin{array}{c}\text { Compréhen- } \\
\text { sion }\end{array}$ & \begin{tabular}{|}
- Interpré \\
-tation
\end{tabular} & $\begin{array}{l}\text { Apprécia- } \\
\text { tion }\end{array}$ & \begin{tabular}{|l|}
$\begin{array}{l}\text { Apport } \\
\text { culturel }\end{array}$ \\
\end{tabular} & Autre & $\begin{array}{c}\text { Discussion } \\
\text { méthodo- } \\
\text { logique }\end{array}$ & \begin{tabular}{|c} 
Exposé \\
magistral
\end{tabular} & \begin{tabular}{|c|}
$\begin{array}{c}\text { Travail } \\
\text { de } \\
\text { groupe }\end{array}$ \\
\end{tabular} & $\begin{array}{c}\text { Cours } \\
\text { dialogué }\end{array}$ & \begin{tabular}{|c|}
$\begin{array}{c}\text { Travail } \\
\text { indiv. }\end{array}$ \\
\end{tabular} & \begin{tabular}{|l|} 
Inter- \\
mèdes
\end{tabular} & \begin{tabular}{|c|} 
Situation \\
de l'objet \\
dans le \\
temps \\
\end{tabular} & \begin{tabular}{|c|} 
Présentifi- \\
cation et \\
élémenta- \\
risation \\
\end{tabular} & $\begin{array}{l}\text { Institu- } \\
\text { tionna- } \\
\text { lisation } \\
\end{array}$ & \begin{tabular}{|c|} 
Evvalua- \\
tion \\
régula- \\
tion
\end{tabular} & $\begin{array}{c}\text { Temps } \\
\text { sans } \\
\text { gestes }\end{array}$ \\
\hline B7-02 & $80 \%$ & $15 \%$ & $0 \%$ & $0 \%$ & $5 \%$ & $0 \%$ & $5 \%$ & $31 \%$ & $50 \%$ & $0 \%$ & $14 \%$ & $0.1 \%$ & $9 \%$ & $5 \%$ & $46 \%$ & $40 \%$ \\
\hline B7-03 & $63,5 \%$ & $26,5 \%$ & $6 \%$ & $0 \%$ & $4 \%$ & $4 \%$ & $1,5 \%$ & $4 \%$ & $88 \%$ & $0 \%$ & $2 \%$ & $1 \%$ & $6,5 \%$ & $1,5 \%$ & $81,5 \%$ & $9,5 \%$ \\
\hline B10-01 & $39 \%$ & $53 \%$ & $5 \%$ & $0 \%$ & $3 \%$ & $12 \%$ & $21 \%$ & $0 \%$ & $63 \%$ & $3 \%$ & $1 \%$ & $3 \%$ & $29 \%$ & $30 \%$ & $37 \%$ & $1 \%$ \\
\hline B10-03 & $36 \%$ & $55 \%$ & $4 \%$ & $5 \%$ & $0 \%$ & $3,5 \%$ & $5 \%$ & $0 \%$ & $89 \%$ & $2 \%$ & $0,5 \%$ & $14 \%$ & $29 \%$ & $4 \%$ & $52 \%$ & $1 \%$ \\
\hline $\mathrm{CH} 7-01$ & $18 \%$ & $23 \%$ & $0 \%$ & $36 \%$ & $23 \%$ & $29 \%$ & $7 \%$ & $0 \%$ & $34 \%$ & $29 \%$ & $1 \%$ & $8 \%$ & $7 \%$ & $10 \%$ & $75 \%$ & $0 \%$ \\
\hline $\mathrm{CH} 7-02$ & $55 \%$ & $24 \%$ & $2 \%$ & $9 \%$ & $10 \%$ & $2 \%$ & $12 \%$ & $0 \%$ & $80 \%$ & $0 \%$ & $6 \%$ & $12 \%$ & $17 \%$ & $5 \%$ & $64 \%$ & $2 \%$ \\
\hline CH10-01 & $69 \%$ & $13 \%$ & $9 \%$ & $6 \%$ & $3 \%$ & $9 \%$ & $14 \%$ & $35 \%$ & $42 \%$ & $0 \%$ & $0 \%$ & $5 \%$ & $7 \%$ & $17 \%$ & $71 \%$ & $0 \%$ \\
\hline $\mathrm{CH} 10-02$ & $39 \%$ & $41 \%$ & $7 \%$ & $11 \%$ & $2 \%$ & $5,5 \%$ & $50,5 \%$ & $0 \%$ & $23 \%$ & $20 \%$ & $1 \%$ & $3 \%$ & $44 \%$ & $29,5 \%$ & $22 \%$ & $1,5 \%$ \\
\hline F7-01 & $55 \%$ & $36 \%$ & $5 \%$ & $0 \%$ & $8 \%$ & $8 \%$ & $2 \%$ & $0 \%$ & $64 \%$ & $23 \%$ & $1 \%$ & $5 \%$ & $36 \%$ & $10 \%$ & $59 \%$ & $0 \%$ \\
\hline F7-02 & $35 \%$ & $57 \%$ & $0 \%$ & $0 \%$ & $8 \%$ & $8 \%$ & $22 \%$ & $2 \%$ & $65 \%$ & $0 \%$ & $3 \%$ & $6 \%$ & $18 \%$ & $20 \%$ & $47 \%$ & $9 \%$ \\
\hline F10-01 & $27 \%$ & $64 \%$ & $5 \%$ & $0 \%$ & $2 \%$ & $2 \%$ & $18 \%$ & $0 \%$ & $71 \%$ & $7 \%$ & $2 \%$ & $3 \%$ & $24 \%$ & $17 \%$ & $56 \%$ & $0 \%$ \\
\hline F10-05 & $36,5 \%$ & $51 \%$ & $1,5 \%$ & $9 \%$ & $2 \%$ & $3,5 \%$ & $23,5 \%$ & $0 \%$ & $69 \%$ & $0 \%$ & $4 \%$ & $3 \%$ & $25 \%$ & $12 \%$ & $57 \%$ & $3 \%$ \\
\hline Q7-01 & $70 \%$ & $0 \%$ & $0 \%$ & $10 \%$ & $20 \%$ & $10 \%$ & $7 \%$ & $0 \%$ & $46 \%$ & $32 \%$ & $5 \%$ & $4 \%$ & $40 \%$ & $9 \%$ & $46 \%$ & $1 \%$ \\
\hline Q10-01 & $47 \%$ & $47 \%$ & $0 \%$ & $0 \%$ & $6 \%$ & $13 \%$ & $4 \%$ & $0 \%$ & $22 \%$ & $60 \%$ & $1 \%$ & $2 \%$ & $17 \%$ & $5 \%$ & $13 \%$ & $63 \%$ \\
\hline
\end{tabular}

27 Ce tableau nous a ensuite permis de procéder à des comparaisons entre les deux niveaux scolaires, puis - à titre purement exploratoire étant donné le nombre réduit des données étudiées à ce sujet - entre les pays. Pour chaque focale, nous nous sommes ainsi demandé dans quelle mesure les activités interprétatives variaient en fonction de l'âge des élèves et, dans une moindre mesure, du contexte scolaire national.

\subsection{La part de l'interprétation dans les séances}

Notre première focale est celle des opérations de lecture. Voici comment celles-ci se répartissent entre les deux niveaux scolaires, dont notre échantillon comportait sept classes par niveau :

Tableau 7. Pourcentage du temps consacré aux opérations de lecture dans les séances : répartition par niveaux

\begin{tabular}{|l|l|l|l|l|l|}
\hline & Compréhension & Interprétation & Appréciation & Apport culturel & Autre \\
\hline Moyenne générale & $48 \%$ & $36 \%$ & $3 \%$ & $6 \%$ & $7 \%$ \\
\hline Niveau 7 (12 ans) & $53,8 \%$ & $26 \%$ & $1,8 \%$ & $7,8 \%$ & $11 \%$ \\
\hline Niveau $10(15$ ans) & $42 \%$ & $46 \%$ & $4,5 \%$ & $4,4 \%$ & $2,5 \%$ \\
\hline
\end{tabular}

29 Ce premier tableau permet de constater quatre tendances significatives :

1. Globalement, l'interprétation occupe plus d'un tiers du temps des séances;

1. Si elle est secondaire par rapport à la compréhension dans les classes de niveau 7 (26\%), cette opération domine dans celles de niveau $10(46 \%)$ : on peut parler à ce propos d'une nette progression entre les deux niveaux;

2. C'est aussi au niveau 10 qu'on se consacre le plus à l'appréciation - trois fois plus qu'au niveau 7 -, même si cette activité reste très limitée $(4,5 \%)^{11}$;

3. En revanche, c'est au niveau 7 qu'on consacre le plus de temps à des apports culturels $(7,8 \%)$ et à des activités autres que les processus de lecture (11\%), c'est-à-dire des interactions liées à la gestion de la classe ou ne relevant pas de l'enseignement de la littérature.

30 Un deuxième tableau nous permet ensuite de comparer la manière dont les opérations de lecture se répartissent, tous niveaux confondus, dans les trois pays pour lesquels 
nous disposions de quatre séances (deux par niveau), à savoir la France, la Belgique et la Suisse ${ }^{12}$.

Tableau 8. Pourcentage du temps consacré aux opérations de lecture dans les séances : répartition par pays

\begin{tabular}{|l|l|l|l|l|l|}
\hline & Compréhension & Interprétation & Appréciation & Apport culturel & Autre \\
\hline France & $38 \%$ & $\mathbf{5 2} \%$ & $1,5 \%$ & $2 \%$ & $5 \%$ \\
\hline Belgique & $\mathbf{5 5 \%}$ & $38 \%$ & $\mathbf{4} \%$ & $1,5 \%$ & $3 \%$ \\
\hline Suisse & $45 \%$ & $25 \%$ & $\mathbf{4 , 5} \%$ & $\mathbf{1 5 \%}$ & $9 \%$ \\
\hline Moyenne générale & $\mathbf{4 6} \%$ & $\mathbf{3 8 , 3} \%$ & $\mathbf{3 , 3} \%$ & $\mathbf{6 , 2} \%$ & $\mathbf{5 , 6} \%$ \\
\hline
\end{tabular}

31 À nouveau, quelques tendances fragmentaires, toutes provisoires compte tenu de la taille de l'échantillon, se dégagent:

1. Les quatre enseignants français consacrent $52 \%$ du temps à l'interprétation, soit le double $\mathrm{du}$ score des quatre enseignants suisses (les quatre enseignants belges se situant exactement dans la moyenne, qui est de $38 \%$ ) ;

1. À l'inverse, la compréhension est privilégiée surtout par les quatre enseignants belges, qui y consacrent $55 \%$ de la séance, soit $17 \%$ de plus que leurs collègues français, et cette fois ce sont les quatre enseignants suisses qui se situent dans la moyenne (45\%);

2. Si l'appréciation est très peu pratiquée globalement, elle l'est trois fois plus dans nos échantillons suisse et belge que dans notre échantillon français ;

3. L'apport culturel apparait huit ou dix fois plus par les enseignants de notre échantillon suisse que par ceux de nos échantillons français et belge.

32 Au-delà de ces constats généraux, si l'on considère de plus près le détail des séances, on constate que la part de l'interprétation y est souvent très difficile à démêler. Nombreux en effet sont les cas d'entrelacements entre compréhension et interprétation, comme dans le passage suivant, extrait de la séance F07-01 :

ENS: Voilà, c'est toujours pour des raisons humanitaires, pour l'humanité, [inaudible] c'est ce qu'il dit à Taratonga, c'est pour une noble cause, pour l'humanité qu'il faut ça. Alors, c'est là-dessus qu'est ma petite question, qu'en pensez-vous et là, vous levez la main?

EL32: Ah! Nul!

ENS : Qu'est-ce que tu en penses Mathilde?

EL7 : Ben, qu'il raconte n'importe quoi?

ENS : Qu'il raconte n'importe quoi, c'est-à-dire?

EL7 : Ben, que, il dit ça mais en fait il veut avoir l'argent.

ENS : Il dit ça mais en fait il veut avoir l'argent.

EL19 : Ben que même si on veut s'en éloigner, tout se rapporte à l'argent.

ENS : Oui, mais, alors, oui, ça, ça serait plutôt la morale de l'histoire. Mais moi ce que je veux savoir, c'est ce que tu penses, est-ce que tu penses qu'il rentre en France pour des raisons humanitaires?

EL19 : Ben non! Les tableaux, il va les vendre.

Ce passage atteste l'ambivalence du verbe "penser » employé par l'enseignant - qui, dans ce contexte, peut signifier aussi bien comprendre qu'interpréter - et du mélange qui en résulte dans les réponses des élèves entre de simples constats, qui relèvent de la compréhension littérale (« en fait il veut avoir de l'argent», «les tableaux, il va les 
vendre »), et la formulation d'hypothèses de signification plus larges, qui relèvent de l'interprétation (" même si on veut s'en éloigner, tout se rapporte à l'argent »).

Nombreux aussi sont les cas d'entrelacements entre interprétation et appréciation. En voici également un exemple, extrait de la séance $\mathrm{CH} 10-02$ :

ENS : Comment on traduit ce personnage ? Il est de mauvaise foi ? Il est naif ? Il est irréprochable? Qu'est-ce que vous en pensez, c'est quoi votre jugement de ce personnage?

[Désigne un élève] Qu'est-ce que vous lisez à propos de ce personnage? Qu'est-ce que vous dites? Vous le jugez comment?

EL2 : Bah, il est... il dit qu'il veut plus affaire avec de l'argent, mais après il... quand il voit les peintures et la possibilité de faire beaucoup d'argent bah il compte l'utiliser. Du coup c'est... euh, il assume pas vraiment son, ce qu'il croit et ce qu'il pense

On le voit, dans ce passage, l'enseignant appelle les élèves à formuler à la fois une interprétation («comment on traduit ce personnage?») et une appréciation («c'est quoi votre jugement?»), mais la réponse de l'élève se situe surtout sur le plan de la compréhension, même si son propos final ( du coup il assume pas vraiment ce qu'il croit et ce qu'il pense ») débouche sur une interprétation.

Par ailleurs, si l'on cherche à identifier la source de l'interprétation dans les échanges, on constate que le plus souvent elle est suscitée par une question ou une consigne de l'enseignant. En témoigne par exemple cet extrait de la séance B07-01:

ENS : Oui, voilà, c'est elle, donc ce ne sont pas des toiles de Gauguin. Donc à ce moment-là, qu'est-ce que le héros constate ?

EL66 : Qu'il s'est fait arnaquer.

ENS : Pourquoi il s'est fait arnaquer?

EL67 : Ben parce qu'il est bête !

ENS : Non, mais pourquoi est-ce qu'il est arnaqué ?

EL68 : À cause de la toile

ENS : Pourquoi est-ce qu'il est arnaqué ?

EL69: Parce que c'est pas la peine [inaudible] parce qu'elle lui a menti.

ENS: Elle lui a menti, c'est vrai... en même temps est-ce qu'elle lui a vraiment menti? Est-ce qu'elle lui a dit "Crois-moi, ce sont des toiles de Gauguin », est-ce qu'elle a dit ça ? Donc, au final elle a pas vraiment menti, elle.

EL70 : C'est un peu l'arroseur arrosé, hein Madame...

Un tel passage illustre bien l'usage systématique et apparemment productif qu'un enseignant peut faire de ce que G. Sensevy (2011, p. 69-74) appelle la "réticence » : un procédé qui consiste à n'accueillir la réponse de l'élève qu'avec des réserves en vue de l'inviter à la préciser et à la développer.

Cela étant, il arrive aussi que l'interprétation soit explicitement prise en charge et débattue entre des élèves. C'est le cas par exemple dans ce passage de la séance B10-01 :

ENS : Et donc si on devait dire quel est le bon et le mauvais dans l'histoire, quel est le bon?

EL66: On ne pourrait pas dire qui est le bon et le mauvais. Le mauvais, c'est la société dans laquelle on vit, parce que... Allez, dans les bons et les mauvais, parce qu'on avait quand même une description très manichéenne au début...

EL67 : Mais lui est bon alors.

ENS : Elle on la met dans quoi ? Les mauvais ? Lève la main.

EL68 : Elle donne une bonne leçon en faisant ça.

ENS : Elle donne une bonne leçon en faisant ça ! Oui ?

EL69 : Elle est mauvaise aussi, elle ment ! Mentir c'est quand même...

ENS : On ne peut pas mentir, c'est vrai... 
EL70: On ne peut pas utiliser la vision manichéenne, parce qu'à un moment on a l'impression qu'elle est bonne, et à un autre, on a l'impression qu'elle est mauvaise... et on peut dire bon, il va se faire arnaquer, mais le narrateur, au début on avait l'impression que c'était le mauvais, mais au moment il se fait arnaquer, c'est lui qui devient le bon à la fin.

ENS : On n'est pas d'accord hein... Bérénice dit l'inverse...

Dans un tel cas, les élèves prennent véritablement l'initiative de l'échange, et l'enseignant cesse d'être le pilote pour devenir en quelque sorte le modérateur de leurs interprétations.

\subsection{Sur quoi portent les interprétations?}

Nous intéressant ensuite à l'objet des interprétations, nous avons répertorié quatorze aspects du texte sur lesquels les enseignants font porter une activité interprétative :

\begin{tabular}{|l|l|l|}
\hline 1. Titre & 6. Personnages principaux & 11. Leçon, morale \\
\hline 2. Thème et champs lexicaux & 7. Relations entre les personnages & 12. Énonciation, ironie \\
\hline 3. Oppositions & 8. Cadre spatio-temporel & 13. Autres éléments linguistiques \\
\hline 4. Schéma narratif & 9. Objets & 14. Valeur \\
\hline 5. Narrateur & 10. Chute & \\
\hline
\end{tabular}

Graphique 1. Pourcentage du temps consacré à l'interprétation des aspects du texte aux niveaux 7 et 10
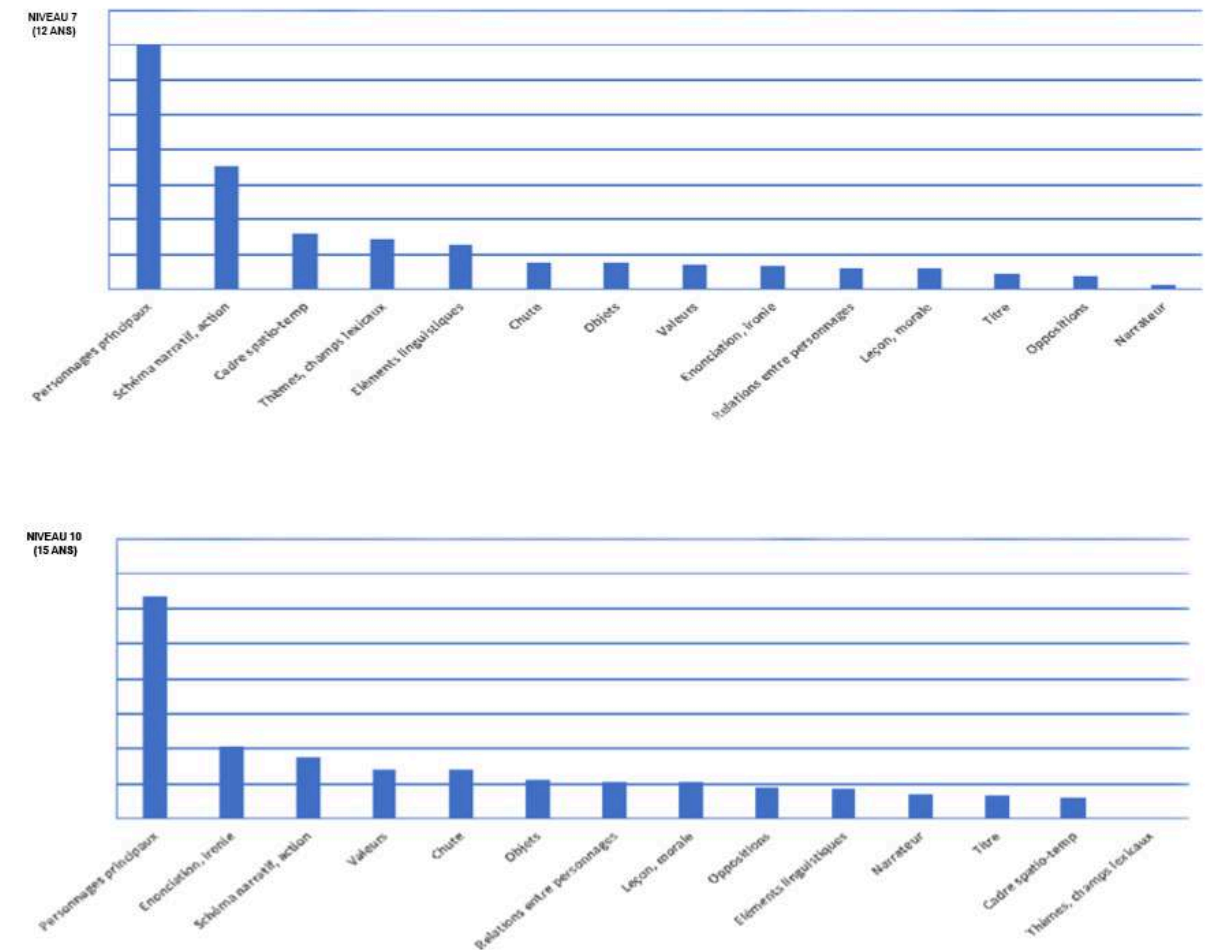

41 Après quantification du temps accordé à chacun de ces aspects (on se réfèrera aux deux tableaux ci-dessus), il apparait que l'activité interprétative se porte prioritairement sur 
les personnages principaux de la nouvelle, et dans une moindre mesure sur le schéma narratif. Des aspects du texte tels que la «chute ", sa "leçon », les «valeurs » portées par le texte, «l'énonciation et l'ironie » ne représentent que $5 \%$ du temps environ et des aspects sont délaissés («thèmes, champs lexicaux »).

Tableau 9. Pourcentage du temps consacré à l'interprétation des aspects du texte : comparaison par niveaux

\begin{tabular}{|c|c|c|c|}
\hline \multicolumn{2}{|c|}{12 ans } & \multicolumn{2}{c|}{15 ans } \\
\hline Personnages principaux & $35 \%$ & Personnages principaux & $32 \%$ \\
\hline Schéma narratif, action & $18 \%$ & Enonciation, ironie & $10 \%$ \\
\hline Cadre spatio-temp & $8 \%$ & Schéma narratif, action & $9 \%$ \\
\hline Thèmes, champs lexicaux & $7 \%$ & Valeurs & $7 \%$ \\
\hline Eléments linguistiques & $6 \%$ & Chute & $7 \%$ \\
\hline Chute & $4 \%$ & Objets & $6 \%$ \\
\hline Objets & $4 \%$ & Relations entre personnages & $5 \%$ \\
\hline Valeurs & $4 \%$ & Leçon, morale & $5 \%$ \\
\hline Enonciation, ironie & $3 \%$ & Oppositions & $4 \%$ \\
\hline Relations entre personnages & $3 \%$ & Eléments linguistiques & $4 \%$ \\
\hline Leçon, morale & $3 \%$ & Narrateur & $4 \%$ \\
\hline Titre & $2 \%$ & Titre & $3 \%$ \\
\hline Oppositions & $2 \%$ & Cadre spatio-temp & $3 \%$ \\
\hline Narrateur & $1 \%$ & Thèmes, champs lexicaux & $0 \%$ \\
\hline
\end{tabular}

Outre la stabilité de l'intérêt porté aux "personnages » et une régression de la part accordée au "schéma narratif ", c'est surtout la progression de certains aspects que l'on observe lorsque l'on passe du niveau 7 au niveau 10. La progression la plus significative concerne "l'énonciation », qui triple sa valeur. Il en va de même pour l'interprétation des « valeurs » portées par le texte, de « la chute » et de la « leçon » ou de la «morale», dont l'addition passe de $11 \%$ à $19 \%$. Pour les aspects cités, la progression est donc sensible du niveau 7 au niveau 10.

43 Ces premiers constats ne doivent pas nous faire ignorer la variabilité, au sein d'un même contexte national, du nombre d'aspects traités d'un point de vue interprétatif dans une même séance et du temps dédié à chacun d'eux.

Graphique 2. La variabilité des interprétations relatives aux aspects du texte : l'exemple des 4 enseignants belges

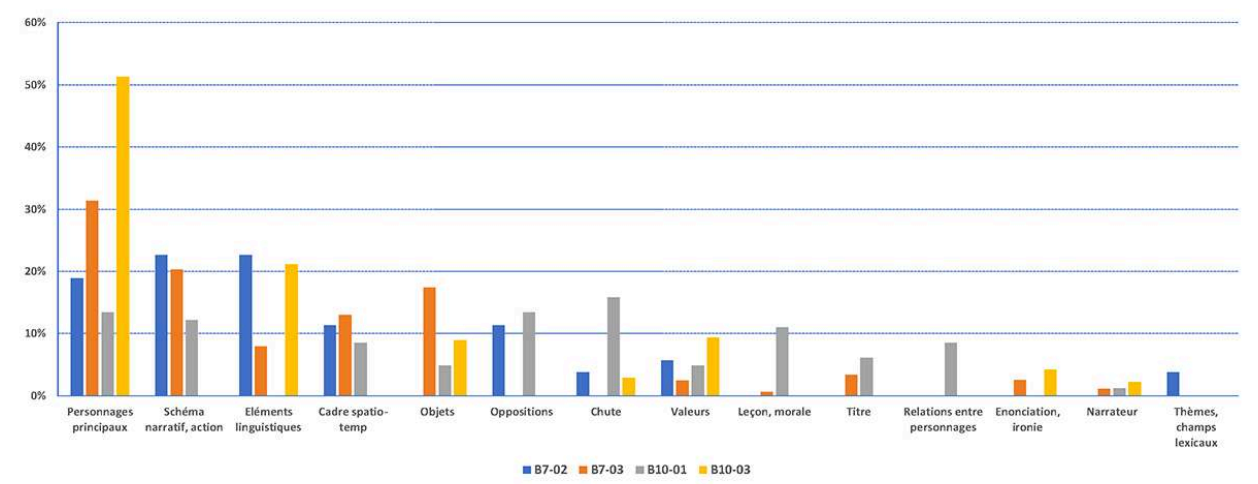



contextualisation de la nouvelle par l'observation de reproductions de toiles de Gauguin $^{13}$, prenant alors le risque que des élèves identifient personnages de la nouvelle et personnages du tableau (ce qui apparait dans un verbatim de niveau 7). Pour cerner l'époque dans laquelle se déroule l'histoire, les professeurs suscitent la production d'inférences à partir d'éléments donnés en notes de bas de page du texte (l'époque est encadrée par les dates de naissance et de mort de Gauguin, la mort de l'auteur, l'existence du franc). Des termes expliqués dans ces mêmes notes de bas de page et questionnées par le professeur pour en vérifier la compréhension (cf. le terme " mercantilisme ») deviennent ensuite des supports pour l'interprétation d'un aspect du personnage. Notons que les références à d'autres textes ou à d'autres personnages 
(Robinson, Picsou/Harpagon, Brel pour les Marquises, Candide), qui sont des réminiscences pertinentes, restent le plus souvent limitées à de simples citations, sans identification réelle des traits communs au narrateur et aux personnages cités.

\section{Chute, morale et valeurs} progression du niveau 7 au niveau 10. Les enseignants mettent bien en évidence le dénouement inattendu de la nouvelle, qu'ils présentent comme une spécificité du genre. Ils rapprochent également cette chute d'autres nouvelles lues en classes ou bien l'opposent aux dénouements d'autres genres littéraires (par exemple, le conte). L'idée d'un " arroseur arrosé » et d'un narrateur qui s'est fait " arnaquer », pour reprendre les propos d'une majorité d'élèves, de niveau 7 comme de niveau 10, peut servir de tremplin à un questionnement sur les enseignements délivrés par la chute. Cependant si les élèves sont en mesure d'exprimer quelques conclusions, comme « il faut se méfier des autres ", " le narrateur s'est menti à lui-même et il s'est fait piéger ", " il ne peut s'en prendre qu'à lui-même ", le plus souvent c'est une interprétation magistrale qui clôt la séance : dans un verbatim de niveau 10 par exemple, le professeur conclut que le narrateur serait le miroir de nous-mêmes, de nos incohérences, de nos désirs, des erreurs auxquelles nous conduisent nos préjugés, et que nous devrions donc avant tout nous méfier de nous-mêmes.

51 Les valeurs que questionne l'œuvre sont approchées à partir de l'observation des agissements du narrateur et des suppositions que le lecteur fait sur ses intentions à partir d'indécisions que ménage le texte. En généralisant, les élèves concluent alors en disant: «On aime tous l'argent », « Il n'y a pas d'innocence ». Cependant on constate que l'identification des «thèmes", aspect très peu traité dans les séances, semble étendre l'interprétation des valeurs du personnage à une réflexion plus générale sur les valeurs (l'argent, son pouvoir, sa fascination; la difficulté à conserver une moralité lorsqu'il est question d'argent ; le choix d'un monde sans argent : possible ou non).

\section{La perception de l'ironie du texte}

52 Pour faire accéder les élèves à l'ironie du texte, les enseignants questionnent prioritairement le titre. Mais là aussi ce sont eux qui apportent des conclusions : on trouve par exemple dans un verbatim niveau 10, "J'ai soif d'innocence » est " la quête d'un paradis perdu » et aussi « un titre très ironique pour l'histoire d'un personnage qui est loin d'être innocent ». Ou encore, dans un autre verbatim de niveau 10, "J'ai soif d'innocence » est "un titre très ironique qui signifie: j'ai soif de l'innocence de l'autre».

Les enseignants peuvent aussi décider de distinguer l'auteur du narrateur, ce qu'ils font assez communément au niveau 7 comme au niveau 10. Mais seuls certains souhaitent aller plus loin que cette distinction et interroger la relation entre narrateur et auteur (le plus souvent au niveau 10). Là aussi ce sont eux qui concluent en disant : l'auteur met en scène un narrateur « se faisant piéger » alors qu'il croit piéger quelqu'un, ce qui confère une dimension ironique à son propos. L'idée que «le texte est tout entier ironique » (expression trouvée dans un verbatim de niveau 10) relève exclusivement de l'interprétation magistrale, et fait écho à la question posée dès le début de la séance : «Quelles sont les intentions de l'auteur lorsqu'il a écrit cela? » L'accès à l'ironie de la 
nouvelle, par le questionnement du titre ou par la distinction entre auteur et narrateur, est donc un pas que les élèves ne franchissent pas seuls. C'est sans doute le lieu où s'exprime le plus la subjectivité de l'enseignant.

La part relative de certains aspects du texte dans l'activité interprétative est donc bien en relation avec le niveau auquel s'adresse le professeur et la gradation dans l'activité interprétative est bien perceptible lorsque l'on passe du niveau 7 au niveau 10. Cela mérite toutefois d'être nuancé dans la mesure où, par exemple, dès le niveau 7 , apparaissent des débats sur les valeurs et les conflits de axiologiques à l'œuvre dans la nouvelle et/ou dans notre société (« une société peut-elle vivre sans argent?») qui orientent les finalités données à l'enseignement de la littérature vers une actualisation du texte.

\subsection{Quels schèmes d'action?}

Au-delà des opérations de lecture et des aspects du texte, les schèmes transversaux constituent un filtre d'analyse intéressant pour porter un regard général sur l'activité en classe. Nous en avons retenu six : l'exposé magistral, le travail de groupe, le cours dialogué, le travail individuel, l'énoncé de consignes et les intermèdes (temps " creux " où les échanges de classe ne portent pas sur le travail en cours).

Dans notre corpus, un schème se distingue nettement : le cours dialogué. Mobilisé $61 \%$ du temps au niveau 7 et $54 \%$ du temps au niveau 10, il constitue la voie privilégiée par laquelle les enseignants travaillent la nouvelle de R. Gary, au contraire du travail de groupe qui n'est en moyenne mis en place que $5 \%$ du temps.

Tableau 10. Pourcentage du temps consacré aux différents schèmes transversaux selon les niveaux scolaires

\begin{tabular}{|l|l|l|l|}
\hline & Niveau 7 & Niveau 10 & Moyenne \\
\hline Enoncé des consignes & $9 \%$ & $7 \%$ & $8 \%$ \\
\hline Cours magistral & $10 \%$ & $19 \%$ & $14.5 \%$ \\
\hline Cours dialogué & $61 \%$ & $54 \%$ & $57,5 \%$ \\
\hline Travail de groupe & $5 \%$ & $5 \%$ & $5 \%$ \\
\hline Travail individuel & $12 \%$ & $13 \%$ & $12.5 \%$ \\
\hline Intermèdes & $4.5 \%$ & $1 \%$ & $5.5 \%$ \\
\hline
\end{tabular}

57 Un scénario récurrent apparait si on se penche sur les passages des verbatims concernant l'interprétation. Les moments interprétatifs commencent souvent par une période plus ou moins longue de cours dialogué et se concluent la plupart du temps par une reprise de la parole de l'enseignant, non plus en tant qu'animateur et distributeur de la parole, mais en tant que « maitre » qui vient synthétiser les éléments de réponse légitimes qui ont surgi au cours de la discussion. L'exemple suivant illustre la succession de ces deux temps :

ENS : Tu disais : oui, il a l'air dans le début de la lecture, sympa et désintéressé... ici ? EL : C'est vrai qu'il a l'air vachement désintéressé, mais le tout, quand je l'ai relu comme ça, ben je me suis dit... [inaudible] 
ENS : Donc ici, comment est-ce qu'on montre - ça rejoint donc ce qu'on a vu précédemment... - que les gens doivent venir vers lui et pas lui vers les gens ? Oui ? EL : Ici il dit [inaudible]

ENS : Donc il fait tout pour son plaisir [inaudible], il faut voir aussi comment l'auteur a écrit ça. Donc a répété euh... de façon tout à fait délibérée euh le déterminant personnel : « mon jardinier, mon pêcheur, mon cuisinier », etc. Voilà. ENS : Donc, de nouveau, tout devra tourner autour de...

EL : Lui...

$\mathrm{P}$ : De Monsieur, voilà. Et alors il dit aussi ce que ce qu'il a cliché, quand il est arrivé sur l'ile, c'est que toute la population, hein c'est ainsi à la fin du paragraphe, toute la population court vers lui... Alors évidemment, dans un cas pareil, c'est un lieu où on a envie de rester... Il est accueilli, mais on voit aussi dans quel état d'esprit il se trouve. Un peu plus loin: «sans bourse délier», mais un peu plus loin il dit «sans bourse délier ", mais aussitôt après : "sur base de l'amitié et de la fraternité la plus simple et la plus touchante et dans le respect mutuel ». (B10-01)

\subsection{Quels gestes didactiques?} tout en permettant un panorama large sur l'activité de classe, il offre une perspective
plus précisément didactique, soulignant la manière dont l'objet enseigné est mis en jeu. Quatre gestes fondamentaux ont été distingués par B. Schneuwly et J. Dolz (2009). La catégorie "situation de l'objet dans le temps" fait référence aux moments où l'enseignant fait appel à la chronologie des apprenants et des apprentissages (mémoire et anticipation). La " présentification/élémentarisation » est une action qui consiste en l'introduction du dispositif via lequel l'objet est traité. L'《institutionnalisation » correspond aux moments de validation des éléments de savoir; l'« évaluationrégulation ", aux échanges de classe et à la modération de ceux-ci par le professeur. Ce dernier geste apparait comme incontournable au sein de notre étude : il représente, en moyenne, plus de la moitié du temps des verbatims traités et est particulièrement dominant au niveau 7 (60\%). La présentification-élémentarisation se trouve en deuxième place, occupant en moyenne $22 \%$ du temps de classe.

Tableau 11. Pourcentage du temps consacré aux différents gestes didactiques selon les niveaux scolaires

\begin{tabular}{|l|l|l|l|}
\hline & Niveau 7 & Niveau 10 & Total \\
\hline Situer l'objet dans le temps & $5 \%$ & $5 \%$ & $5 \%$ \\
\hline Présentifier/élémentariser & $19 \%$ & $25 \%$ & $22 \%$ \\
\hline Évaluer/réguler & $60 \%$ & $44 \%$ & $52 \%$ \\
\hline Institutionnaliser & $9 \%$ & $16 \%$ & $12,5 \%$ \\
\hline
\end{tabular}

Notons que, à nouveau, les moments interprétatifs s'organisent souvent autour d'une sorte de scénario mettant en scène les gestes suivants : à la suite de l'introduction du dispositif (présentification-élémentarisation), le professeur lance un échange qu'il régule et évalue. Celui-ci est clôt (ou du moins, ponctué) par des pauses « institutionnalisantes » où sont validés, via différentes techniques, les éléments dignes d'être retenus par les élèves. Ce scénario n'est pas sans rappeler celui qui a été suggéré dans le point précédent. Doit-on voir là un certain figement du mode de 
communication entre élèves et professeur? Quoi qu'il en soit, il est certain que l'activité interprétative, même si elle tend à toujours être entérinée par l'autorité enseignante, ne fait que très rarement fi de la parole de l'élève. L'interprétation d'un texte serait donc avant tout un objet à discuter, dans une sorte de perspective inductive.

ENS : A Taratonga. C'est le personnage féminin, [inaudible]. Alors, revenons à, aux traces d'égoïsme, vous m'avez cité «mon pécheur, la présence du “je", mon pécheur, mon jardinier, mon cuisinier». Est-ce que vous, si on était en commentaires, qu'est-ce qu'on pourrait exploiter pour montrer à quel point ce narrateur peut paraitre, vous m'avez dit, égoïste et profiteur?

EL21 : Qu'il, ben, qu'il dit « mon » à chaque fois et c'est comme si, enfin, c'était à lui et à personne d'autre.

ENS : Oui, d'accord, il y a une notion de?

EL8 : De possession.

ENS : D'appartenance, de?

EL8 : Possession.

ENS : Possession, alors, possession, la possession, alors, appartenance, presque de propriété, alors que, justement, c'est quelque chose qui est dénoncé. Déduit ellemême Euh, vous pouvez identifier le, grammaticalement, la nature du mot, placé devant un nom ? Pardon Kim?

EL21 : Déterminant.

ENS : D’accord, donc le déterminant possessif. Euh, il est placé, euh, par quel indice, euh, l'auteur nous fait-il sentir, justement, ce côté, dans l'écriture, ce côté égoïste et profiteur là, "mon pécheur, mon cuisinier, mon jardinier ", quel est l'outil [inaudible] ? Manon?

EL11 : Une énumération.

ENS : Ben, oui, une énumération. Une répétition.

EL3 : Une accumulation.

ENS : Une accumulation, voilà. Donc, en accumulation, donc on voit bien, on perçoit bien à quel point le narrateur, vous l'avez vu, était nourri d'un désir de possession. «Sans bourse délier » donc il se crée, oui, tu m'as parlé aussi tout à l'heure, tu as dit Thomas, il les nomme comme du bétail, précise?

EL7 : Ben, il a aucune considération euh réelle pour eux, quoi, il les appelle comme si c'était juste une population dont il allait se servir pour son propre intérêt.

ENS : D'accord, donc une attitude de mépris. Oui Clément?

EL5 : Il dit quelques centaines de têtes.

ENS : Voilà.

EL5 : C'est péjoratif.

ENS: C'est péjoratif, d'accord. Une attitude de mépris on le note. Tu voulais rajouter quelque chose, je t'ai coupé ? [Pas de réponse de l'EL]. C'est péjoratif, c'est très bien, comme du bétail. Oui, d'accord. (F10-01)

\subsection{Quels genres d'activités scolaires?}

60 Nous nous sommes enfin demandé quels genres d'activités scolaires (ou «GAS ») de lecture de la littérature les enseignants privilégiaient lorsqu'ils travaillaient l'interprétation avec leurs élèves.

61 Une première analyse, générale, consiste à identifier si une convergence peut être établie entre les activités interprétatives dans la séance et certains genres d'activités de lecture. 
Tableau 12. Comparaison du pourcentage du temps consacré aux interprétations et aux genres d'activités scolaires

\begin{tabular}{|c|c|c|c|c|c|c|c|c|}
\hline & FOCALES & Opération & \multicolumn{6}{|c|}{ GAS les plus significatifs } \\
\hline & Critères & $\begin{array}{l}\text { Interpré- } \\
\text { tation }\end{array}$ & $\begin{array}{c}\text { Explication } \\
\text { de texte }\end{array}$ & $\begin{array}{l}\text { Travail de la } \\
\text { compréhension }\end{array}$ & $\begin{array}{l}\text { Études de } \\
\text { dimensions } \\
\text { de l'objet }\end{array}$ & $\begin{array}{l}\text { Débat } \\
\text { inter- } \\
\text { prétatif }\end{array}$ & $\begin{array}{l}\text { Mise en } \\
\text { réseaux }\end{array}$ & $\begin{array}{c}\text { Production } \\
\text { de textes }\end{array}$ \\
\hline \multirow[t]{3}{*}{ MOYYENNES } & $\begin{array}{l}\text { Moyenne } \\
\text { niveau } 7\end{array}$ & $26 \%$ & $43 \%$ & $10 \%$ & $6 \%$ & $6 \%$ & $3 \%$ & $2 \%$ \\
\hline & $\begin{array}{l}\text { Moyenne } \\
\text { niveau } 10\end{array}$ & $46 \%$ & $47 \%$ & $13 \%$ & $8 \%$ & $4 \%$ & $8 \%$ & $9 \%$ \\
\hline & $\begin{array}{l}\text { Moyenne } \\
\text { générale }\end{array}$ & $36 \%$ & $45 \%$ & $7 \%$ & $7 \%$ & $5 \%$ & $6 \%$ & $5 \%$ \\
\hline \multirow{3}{*}{$\begin{array}{c}\text { CLASSES } \\
\text { REMARQUABLES }\end{array}$} & F10-05 & $51 \%$ & $53 \%$ & $5 \%$ & $19 \%$ & $7 \%$ & $31 \%$ & $0 \%$ \\
\hline & F10-01 & $64 \%$ & $44 \%$ & $16 \%$ & $0 \%$ & $9 \%$ & $14 \%$ & $0 \%$ \\
\hline & B10-01 & $53 \%$ & $44 \%$ & $12 \%$ & $0 \%$ & $0 \%$ & $0 \%$ & $0 \%$ \\
\hline
\end{tabular}

Dans les 14 séances de notre corpus, le GAS le plus représenté est celui de l'«explication de texte", et l'on n'identifie pas, de manière probante, une surreprésentation de ce GAS dans les séances qui accordent une plus grande place aux activités interprétatives. Au-delà de ce seul GAS, on identifie, lorsqu'on regarde attentivement les séquences interprétatives des verbatims, un phénomène d'association de trois genres: l'«explication de texte», la "régulation de la compréhension » et la « lecture à haute voix ». Cette combinaison, qui joue souvent au niveau micro dans les séances, dessine un micro-scénario reproduit tout au long des séances: on lit un passage précis, délimité par l'enseignant, puis celui-ci guide les élèves, en s'appuyant sur le texte, vers une interprétation associée aux techniques de l'explication de texte (repérage et interprétation de procédés, relevés interprétés). Parfois, mais plus rarement, ce sont les élèves qui sont à l'initiative d'une séquence interprétative, car ils sollicitent un approfondissement. Dans ce cas, l'enseignant pointe un segment textuel qu'il relit avec les élèves avant de revenir sur leur interprétation :

ENS : Oui hein il recherche la paix de l'esprit, les gens simples, serviables, euh, le désintéressement [ENS écrit au tableau] alors "tu me parles d'êtres simples et serviables » qui a donné cette citation? [Un élève lève la main]. Oui, regarde ce qu'il $\mathrm{y}$ a juste après. Continue de lire ce qu'il y a juste après.

EL 32 : Au cœur complètement incapable de calculs sordides.

ENS : Oui, continue.

EL32: Auxquels je pourrais tout demander en accordant mon amitié en échange, sans crainte de quelque mesquine considération [inaudible].

ENS : Oui ça rejoint ce que dis Mathis, finalement ce qu'il recherche c'est des gens simples serviables incapable de faire des calculs qu'il appelle des calculs sordides hein euh et auxquels il pourrait tout demander hein sans rien avoir à donner en échange. Qu'est-ce que ça montre quand même, qu'est-ce qu'il garde en tête ? EL33 : Bah il veut que ce soit le roi que ce soit lui qui peut gouverner.

ENS : Oui qu'est ce qui l'arrange dans cette innocence là qu'il recherche?

EL2 : ça l'arrange qu'il n'ait rien à payer et qu'il est tout en échange.

ENS : Oui.

EL34 : C'est un peu comme dans l'oncle Picsou il est avare.

ENS : Oncle Picsou ! Oui alors en fait on a l'impression que ça l'arrange dans un sens que ça va lui permettre d'obtenir des choses à moindre frais. (F07-01) ${ }^{14}$

63 Comme on le voit, l'enseignant ajuste l'interprétation spontanée d'un élève en demandant au groupe de poursuivre sa lecture car le passage suivant se prête à une révision de l'interprétation première. Ses questions orientent fermement 
l'interprétation des élèves qui parviennent à cerner le caractère profiteur du personnage, notamment en se référant au personnage de Picsou.

Notons également que la mise en réseau et l'étude de dimensions de l'objet sont des GAS qui permettent aux enseignants de développer des séquences interprétatives productives. L'enseignant prévoit alors d'apporter des savoirs - ou de les réactiver - sur telle référence ou tel objet, car leur mobilisation peut enrichir l'interprétation. Celle-ci est alors souvent très guidée par le professeur, comme cet exemple le montre :

ENS : Eh oui, voilà, c'est paradisiaque! Alors, paradisiaque [ENS écrit], Ok. [Quelques secondes de brouhaha] Le paradis, le paradis c'est le monde, est-ce que vous pouvez mettre maintenant en lien "paradis » et « innocence»? Qu'est-ce que vous savez du paradis?

E17 : C'est le jardin d'Éden.

ENS : C'est le jardin d'Éden, alors, c'est-à-dire?

E17 : Ben euh.

ENS : C'est quoi le jardin d'Éden ? Y'en a peut-être qui ne savent pas ce que c'est que le jardin d'Éden. (F10-02)

Enfin, nous remarquons que les genres de l'«expression d'avis sur le texte » ou du «débat interprétatif» sont peu sollicités par les enseignants, y compris ceux qui consacrent beaucoup de temps à l'interprétation. Ainsi semble-t-il que, dans la moyenne de nos 14 séances, les pratiques d'enseignement de l'interprétation prennent appui sur des genres traditionnellement ancrés dans les formes scolaires (la lecture à haute voix ou l'explication de texte) plutôt que sur des genres didactiques ayant été conçus plus récemment (le débat interprétatif) et sollicitant l'implication subjective des élèves (avis sur le texte, débat interprétatif).

Pour aller plus loin dans l'analyse sur le plan qualitatif, nous avons étudié les différentes séquences interprétatives associées aux GAS dans les verbatims.

Tout d'abord, nous repérons peu d'écart entre le choix des genres dédiés à l'interprétation pour les élèves de niveau 7 et pour les élèves de niveau 10. Notons également que les enseignants qui développent le plus d'activités interprétatives sont ceux qui utilisent la variété la plus importante de GAS (jusqu'à 6 différents) : tandis que «l'explication de texte» couvre une grande part de l'activité interprétative des séances, associée à la lecture et parfois à la régulation de la compréhension, comme nous l'avons précisé plus haut, l'appel aux «réseaux», à l'«avis personnel», au "débat », au "traitement d'un objet spécifique » ou au "discours thématique » sont utilisés tour à tour. Par exemple, dans l'une des classes mobilisant le plus l'interprétation, la classe française F10-05, l'enseignante travaille plus de $30 \%$ du temps sur la sollicitation de réseaux. Ajoutons que les genres "production» ou «avis sur le texte " semblent particulièrement propices aux fins de séance quand elles sont interprétatives, comme si un trajet interprétatif se réalisait, à travers l'utilisation des différents GAS : l'« explication de texte » ou la « compréhension » permettrait d'étayer de manière plus collective mais aussi plus magistrale un accès à l'interprétation que la fin de la séance permettrait de personnaliser ou de rendre plus autonome. Au contraire, dans certaines séances, c'est l'enseignant qui conclut en synthétisant l'interprétation que le travail d'explication de texte a conduit à privilégier.

ENS : Alors, juste pour terminer, je voudrais vous demander d'essayer d'imaginer une suite à cette histoire. À votre avis ? Anaïs ? Qu'est-ce que à votre avis va faire le narrateur? Est-ce qu'il va rester là, est-ce qu'il va rentrer en France? Fatima? (B07-02) 
ENS : On voit elle est totalement indifférente au narrateur qui veut prendre ces toiles peintes, puis à la fin on apprend qu'en fait elle joue, elle joue avec ça. Donc c'est une nouvelle qui... qui pourrait [inaudible, élèves commencent à ranger leurs affaires] montrer que tout le monde est mis dans le même, enfin tout le monde est mis dans le même paquet. (CH10-01)

Enfin, même si l'aspect réduit de notre échantillon ne permet pas d'analyse liée au contexte national, nous remarquons que, dans notre corpus, le genre du débat est quasi absent des classes françaises tandis qu'il est bien plus fréquent dans les classes belges et suisses. L'élargissement de notre étude nous permettra de vérifier cette tendance sur laquelle l'étude de cas attire l'attention.

Pour conclure sur le volet «enseignement de l'interprétation » dans les 14 séances de notre corpus, nous soulignerons quelques constats saillants. En premier lieu, si la place des séquences interprétatives dans la séance évolue très significativement entre les niveaux 7 et 10, cette évolution ne se marque pas forcément par des conduites différentes dans les schèmes transversaux, les gestes didactiques ou les genres d'activités scolaires utilisés par les enseignants. Ceux-ci sont en effet très similaires dans les deux niveaux de scolarisation. Par ailleurs on identifie des pratiques majoritaires relativement traditionnelles : dans les séquences interprétatives, le travail est concentré sur les personnages, organisé en cours dialogué, selon une conduite de classe privilégiant les régulations et très largement dominée par des activités d'explication de texte. De plus, dans ces pratiques, l'enseignant occupe une grande place, tandis que les activités qui conduiraient à des interprétations plus autonomes ou plus subjectives, comme le schème du travail de groupe ou individuel ou le GAS du "débat interprétatif ", restent peu mobilisées. Enfin, on pourrait aussi se demander si la durée limitée de la séance n'a pas constitué un frein pour les approches plus novatrices, qui s'inscrivent peut-être plus aisément dans une plus longue durée...

\section{Premières conclusions et pistes de rapprochements}

$70 \mathrm{Au}$ terme de cette longue analyse, il convient de rappeler les limites méthodologiques de notre double enquête : l'échantillon de 377 questionnaires et 16 séances, même si cela constitue déjà un ensemble de données appréciable, reste insuffisant pour formuler des généralisations. Tout au plus pouvons-nous observer des tendances compte tenu de la saturation déjà apparente des résultats.

71 Par ailleurs, nous n'ignorons pas les difficultés que les élèves les plus faibles ont éprouvées à répondre au questionnaire en raison de la complexité du texte et des contraintes liées au contexte de passation de l'épreuve. En répondant à ce questionnaire, les élèves ont-ils vraiment rendu compte de leur compétence lectorale ? Il est interpelant en tout cas de constater que, si ce questionnaire servait à évaluer des apprentissages, près du tiers des élèves de 12 ans et près du quart des élèves de 15 ans $\mathrm{y}$ échoueraient. Cela tient-il seulement à un défaut de littératie de leur part ou à la difficulté que présente l'outil du questionnaire de lecture, ou encore au temps limité dont ils disposaient pour y répondre? Face à cette situation, le rôle des didacticiens nous semble être de tenter d'expliquer les interprétations, notamment en considérant les ressources subjectives convoquées.

72 En outre, plusieurs questions d'ordre didactique se posent à la suite de nos constats. Aurions-nous obtenu des réponses très différentes si nous avions interrogé les élèves 
sur ce que le texte leur dit, plutôt que sur ce que l'auteur cherche à leur dire ${ }^{15}$ ? Comment enseigner la justification, afin de les amener à interpréter avec plus de nuances? Faut-il accorder plus d'espace au sujet-lecteur et l'encourager à recourir à une variété de ressources subjectives, et ce, plus tôt dans le parcours scolaire ? Certes, il s'agit là de questions d'ordre plus prescriptif (que faut-il enseigner?) que scientifique (que se passe-t-il dans la classe ?), mais elles sont cruciales pour les didacticiens, et un des buts de notre recherche est de pouvoir apporter quelques éclairages utiles à leur propos.

73 Cela étant, il convient aussi à présent de se demander dans quelle mesure notre analyse des pratiques enseignantes peut s'articuler avec celle des réponses des élèves aux questionnaires.

74 Pour répondre à cette question, nous pouvons d'abord mettre en relation la place de l'interprétation dans les séances et les compétences interprétatives mobilisées par les élèves. Nous constatons à ce propos une évolution parallèle : l'importance et la richesse des réponses des élèves évoluent entre les niveaux 7 et 10 de manière convergente avec la place croissante accordée à l'interprétation par leurs enseignants. Certes, nous l'avons souligné précédemment, il parait naturel que les élèves du niveau 7 rencontrent plus de difficulté à interpréter un texte résistant que les élèves du niveau 10. De même, les enseignants qui consacrent une part importante de la séance à accompagner la compréhension du texte accordent un temps plus réduit à l'interprétation. Ajoutons que les élèves du niveau 7 manquent sans doute de référents externes et d'une certaine maturité pour accéder à certaines interprétations, ce qui peut expliquer que leurs enseignants n'abordent pas les aspects qu'ils jugent moins accessibles.

75 Nous observons également des convergences entre les aspects du texte privilégiés par les élèves dans les questionnaires et ceux que privilégient les enseignants: dans les deux cas, les personnages, les valeurs et la leçon du texte constituent les lieux interprétatifs principaux, et ce dans les deux niveaux.

76 La mise en relation des questionnaires et des conduites de classes fait apparaitre d'autres éléments qui peuvent nourrir notre réflexion sur certaines difficultés des élèves. En l'occurrence, notre analyse des réponses au questionnaire souligne le nombre important de réponses peu acceptables et d'interprétations non justifiées, en particulier du niveau 7. Ces difficultés semblent mettre en évidence le manque d'autonomie des élèves. Or, nous avons pu montrer que les séquences interprétatives des leçons sont très guidées par les enseignants, que ce soit sous la forme du cours dialogué ou de l'exposé magistral, ou encore par des pratiques de découpage du texte en petites unités ou de forte régulation. Ces pratiques ne laissant que peu de place à l'autonomie et à la réflexion propre de l'élève, celui-ci peut se trouver démuni lorsqu'on lui demande d'interpréter le message du texte ${ }^{16}$ sans autre soutien car la vision globale que cette opération nécessite peut alors être effacée par la segmentation du traitement textuel.

77 Enfin, nous pouvons rapprocher les réponses des élèves et les genres d'activités scolaires pratiquées par les professeurs. Nous l'avons noté, les séances les plus riches en activités interprétatives sont celles qui mobilisent une plus grande diversité de GAS. Ces séances concernent-elles les classes où les réponses aux questionnaires présentent la plus grande richesse de pistes interprétatives? Cette piste doit être étudiée de près. De même, il semblerait - mais cela doit être confirmé - que, dans les classes où se pratique davantage la production de «textes du lecteur » (débat interprétatif, avis sur 
le texte), les justifications apportées par les élèves dans les questionnaires sont plus développées.

Différents types de relations peuvent donc être envisagés entre nos deux ensembles de données. Ils permettent de mettre en évidence des liens possibles entre des pratiques enseignantes et les compétences interprétatives des élèves mais aussi l'adaptation de certains enseignants aux compétences et aux difficultés réelles de leurs élèves, comme nous l'avons identifié en repérant l'évolution de l'importance accordée par les enseignants à la compréhension et à l'interprétation entre les classes de niveau 7 et de niveau 10.

Pour conclure ce bilan, nous pouvons dégager quelques implications de notre étude pour la réflexion didactique. Il apparait tout d'abord assez clairement que les activités interprétatives enrichissent la séance et permettent aux élèves de manifester la polysémie de la nouvelle de R. Gary. À ce propos, il semble que les classes où les activités interprétatives proposées par les enseignants sont les plus variées, que ce soit en termes de choix didactiques ou en termes d'aspects du texte, sont aussi celles où les élèves aboutissent aux interprétations les plus riches, tant dans les séances que dans les questionnaires, ce qui laisse supposer que certains enseignants avaient déjà exercé leurs élèves à cette compétence avant la passation du questionnaire.

Enfin, nous remarquons que les deux GAS spécifiquement dédiés à l'interprétation que sont le débat interprétatif et l'expression d'avis sur le texte sont très peu mis en œuvre dans notre corpus. Cela nous incite à poursuivre l'enquête, d'une part en nous intéressant aux pratiques de formation dont les enseignants ont bénéficié, d'autre part en vérifiant la prégnance des formes scolaires traditionnelles auxquels ces deux genres n'appartiennent pas.

\section{BIBLIOGRAPHIE}

AEBY DAGHÉ, S. (2014). Candide, la fée carabine et les autres. Vers un modèle didactique de la lecture littéraire. Bruxelles/Berne/Berlin : P. Lang. En ligne : https://www.peterlang.com/view/title/ 36164.

BRUN-LACOUR, I. \& DUFAYS, J.-L. (à paraitre). « De la classe à la recherche et de la recherche à la classe, quelle circulation des savoirs ? Observations et analyses au départ du projet "Gary" ».

Transpositio. Littérature et enseignement 3. En ligne : https://www.transpositio.org/.

BRUNEL, M., DUfAYS, J.-L., et ÉMERY-BRUNEAU, J. (2017). « Lire un même texte littéraire de 12 à 15 ans : quels apprentissages et quels dispositifs ? Regards croisés France, Belgique, Québec ». In : Brunel, M. et al. (dirs.). L'Enseignement et l'apprentissage de la lecture aux différents niveaux de la scolarité.

Namur : Presses universitaires de Namur, p. 137-162.

BRUNEL, M. et al. (2018). « Le discours des élèves sur les valeurs du texte littéraire et leur exploitation didactique par les enseignants : quelles variations selon les classes d'âge et selon les pays ? ». In : Rouvière, N. (éd.). Enseigner la littérature en questionnant les valeurs. Bruxelles/Berne/ Berlin : P. Lang, p. 279-302. 
BRUNEL, M. et al. (à paraitre, 2021). Lire en classe l'altérité : quelle progression entre 12 et 15 ans ? Deux analyses à partir de la nouvelle J'ai soif d'innocence de Romain Gary. Namur : Presses universitaires de Namur.

CHEVALLARD, Y. (1985). La Transposition didactique. Grenoble : La pensée sauvage.

DUFAYS, J.-L. (2010). « Discontinuités dans l'enseignement de la littérature en Belgique francophone ». Le français aujourd'hui 168, p. 33-42. En ligne : https://www.cairn.info/revue-lefrancais-aujourd-hui-2010-1-page-33.htm.

DUMORTIER, J.-L., VAN BEVEREN, J. \& VRYDAGHS, D. (dirs) (2012). Curriculum et progression en français. Actes du $11^{e}$ colloque de l'AIRDF (Liège, 26-28 août 2010). Namur : Presses universitaires de Namur.

GARY, R. (2012) [1962]. J'ai soif d'innocence. Paris : Larousse.

HÉBERT, M. (2013). « Lire, commenter, discuter un même roman au primaire et au secondaire : quelles différences ? ». Revue des sciences de l'éducation 39 (1), p. 119-146. En ligne : https:// doi.org/10.7202/1024535ar.

JORRO, A. \& CROCE-SPINELLI, H. (2010). « Le développement de gestes professionnels en classe de français. Le cas de situations de lecture interprétative ». Pratiques 145-146, p. 125-140. En ligne : https://journals.openedition.org/pratiques/1527.

LARRIVÉ, V. (2015). « Empathie fictionnelle et écriture en “je” fictif ». Repères 51, p. 157-176. En ligne : https://journals.openedition.org/reperes/913.

MARLAIR, S. \& Dufays, J.-L. (2008). « Quels gestes dans la classe pour quel enseignementapprentissage de la littérature ? Regard sur quatre leçons de $5^{\mathrm{e}}$ année secondaire ». In : Bucheton, D. \& Dezutter, o. (dirs). Le Développement des gestes professionnels dans l'enseignement du français. Un défi pour la recherche et la formation. Louvain-la-Neuve : De Boeck, p. 61-82.

RIC@UR, P. (1990). Soi-même comme un autre. Paris : Éditions du Seuil.

RONVEAUX, C. \& SCHNEUWLY, B. (2018) (dirs). Lire des textes réputés littéraires : disciplination et sédimentation. Enquête au fil des degrés scolaires en Suisse romande. Bruxelles/Berne/Berlin : P. Lang. SAUVAIRE, M. (2013). Diversité des lectures littéraires : comment former des sujets lecteurs divers ?. Thèse en didactique : Université Laval/Université de Toulouse Le Mirail. En ligne : https:// corpus.ulaval.ca/jspui/bitstream/20.500.11794/24045/1/29976.pdf.

SCHNEUWLY, B. \& DOLZ, J. (dirs.). (2009). Des objets enseignés en classe de français. Rennes : Presses universitaires de Rennes.

SENSEVY, G. (2011). Le Sens du savoir. Éléments pour une théorie de l'action conjointe en didactique. Louvain-la-Neuve : De Boeck.

THOMPSON, J. (1987). Understanding teenagers' reading. New York : Nichols Publishing Company.

\section{NOTES}

1. Nouvelle publiée originellement dans le recueil Gloire à nos illustres pionniers, Gallimard, 1962, et republiée en 2012 par Larousse dans une version réduite de ce recueil intitulée J'ai soif d'innocence et autres nouvelles à chute.

2. Précisons que ces 16 classes n'ont fait l'objet que de 14 séances différentes car les quatre classes québécoises ne sont associées qu’à deux enseignants qui ont animé 
chacun deux séances selon des scénarios identiques, ce qui ne permet pas de les traiter comme des données distinctes.

3. Nous avons écarté la sixième catégorie de M. Sauvaire, qui concerne le rapport aux outils, aux supports et au temps et à l'espace dans lequel se déroule la lecture (ressources matérielles et spatiotemporelles), car nous n'en avons repéré aucune occurrence dans nos données. Par ailleurs, nous essaierons de montrer dans notre analyse des données comment la construction de différents sens à l'aide d'indices du texte s'articule avec les ressources des sujets lecteurs.

4. En particulier, nous avons distingué quatre types fondamentaux d'appréciation, qui portent respectivement sur la cognition (l'élève apprécie ou non la clarté qu'il attribue au texte), sur la dimension éthique (l'élève apprécie ou non les comportements des personnages), sur la dimension référentielle (l'élève apprécie ou non le type d'univers proposé par le texte) et sur la dimension esthétique (l'élève apprécie ou non la manière dont le texte est construit et capte son attention et/ou ses émotions).

5. Dans nos premiers travaux, nous avions étudié les trois " genèses » développées par Y. Chevallard (1985) et G. Sensevy (2011), à savoir la chronogenèse, qui concerne le déroulement linéaire de la leçon, la topogenèse, qui concerne la distribution des rôles au sein de la classe, et la mésogenèse, qui concerne la gestion par l'enseignant du milieu didactique, c'est-à-dire des dispositifs.

6. Dans la partie "interprétations formulées sur le texte», le pourcentage indiqué correspond au nombre d'interprétations rapporté au nombre d'élèves, compte tenu du fait que certains élèves proposent plusieurs interprétations.

7. Ce «niveau recommandé » est mentionné par l'éditeur sur la $4^{\mathrm{e}}$ de couverture sans justification.

8. Nous aurions pu réaliser des entretiens avec quelques élèves pour confirmer cette hypothèse.

9. Pour ce faire, nous procédons à un classement en catégories de chaque focale choisie en appliquant un code couleurs sur les verbatims, puis nous réalisons des négociations interjuges afin de croiser et d'accorder deux regards sur un même codage. Enfin, nous reportons en pourcentage de temps, pour chaque leçon, la part correspondant à chaque aspect d'une grille traitée.

10. Les enseignants y sont identifiés d'abord par l'initiale de leur pays $(B, C H, F, Q)$ puis par le niveau scolaire (7 ou 10).

11. Ce constat confirme celui que nous avons formulé lors d'une présente analyse sur la base d'un corpus plus limité (8 classes, 2 par pays) (Brunel et al., 2018).

12. Parler du Québec ici n'aurait pas eu de sens puisque, pour ce pays, nous ne disposions que d'une classe par niveau.

13. Dans un verbatim d'une classe française, le cours prend alors le tour d'une séance d'histoire des arts.

14. Les passages en gras sont identifiés comme relevant de l'interprétation.

15. Notre objectif étant de mesurer le rapport des élèves aux "droits du texte », nous avions formulé notre $3^{\mathrm{e}}$ question dans un sens intentionnaliste (" selon toi, que cherche à nous dire l'auteur? ») qui laissait peu de place aux « droits du lecteur ».

16. Ce qui est l'objet de la question 3 du questionnaire. 


\section{RÉSUMÉS}

Dans cet article qui s'inscrit dans le cadre d'un projet de recherche en cours, nous nous attachons à analyser, d'une part la manière dont 377 élèves issus de 16 classes belges, françaises, québécoises et suisses des niveaux scolaires 7 et 10 (12 et 15 ans) lisent une même nouvelle de Romain Gary, et d'autre part, la manière dont leurs enseignants exploitent ensuite librement le même texte durant une séance en classe. Le premier volet, qui porte sur les compétences interprétatives des élèves dans leurs réponses à un questionnaire, met en évidence la progression qui se manifeste entre les deux niveaux, tant en ce qui concerne la qualité des interprétations que leur nombre. Nous nous intéressons ensuite à l'activité des enseignants, en étudiant d'abord la part que le travail interprétatif y occupe par rapport aux autres processus de lecture, puis ses relations avec différents schèmes d'action, différents genres d'activité scolaires et différents gestes professionnels, et nous nous demandons dans quelle mesure il varie en fonction de l'âge des élèves et du contexte national. Enfin, nous nous interrogeons sur les liens qui peuvent être établis entre nos deux ensembles de données : dans quelle mesure les enseignants exploitent-ils les compétences et les difficultés interprétatives des élèves révélées par les questionnaires? Et dans quelle mesure, en retour, les réponses des élèves aux questionnaires paraissent-elles influencées par des démarches que privilégient leurs enseignants?

In this article, which is part of an ongoing research project, we aim to analyze on the one hand the way in which 377 pupils from 16 Belgian, French, Quebec and Swiss classes in grades 7 and 10 (12 and 15 years old) read the same story of Romain Gary, and on the other hand the way their teachers freely exploit the same text during a classroom session. In the first part, which focuses on students' interpretative skills in their answers to a questionnaire, we highlight the progression that occurs between the two levels, as regards both the quality of the interpretations and their number. We are then interested in the activity of the teachers. We study successively the part that the interpretative work occupies there in relation to other reading processes, then the links with different schemes of action, kinds of school activities and professional gestures, and we wonder to what extent it varies according to the age of the pupils and to the national context. Finally, we question the links that can be established between our two sets of data: to what extent do teachers exploit the interpretative skills and the difficulties of the pupils revealed by the questionnaires? And to what extent, in turn, can student responses to the questionnaires appear to be influenced by approaches that are favored by their teachers?

\section{INDEX}

Mots-clés : lecture, littérature, compétences, interprétation, pratiques enseignantes, gestes didactiques, genres d'activités scolaires

\section{AUTEURS}

\section{JEAN-LOUIS DUFAYS}

Université catholique de Louvain, Laboratoire Cripedis, B-1349 Louvain-la-Neuve, Belgique

\section{MAGALI BRUNEL}

Université Côte d'Azur, Line/Inspé, F-06108 Nice, France 


\section{JUDITH ÉMERY-BRUNEAU}

Université du Québec en Outaouais, CRIFPE, QC J8X 3X7 Gatineau, Canada

\section{MARIE BARTHÉLEMY}

Université catholique de Louvain, Laboratoire Cripedis, B-1349 Louvain-la-Neuve, Belgique VINCENT CAPT

Université de Genève/HEP de Vaud, Grafe, S-1205 Genève, Suisse

\section{ISABELLE BRUN-LACOUR}

Université Clermont Auvergne/ESPE Clermont-Auvergne, F-63000 Clermont-Ferrand, France SONYA FLOREY

HEP de Vaud, LPIC, S-1014 Lausanne, Suisse

MARTIN LÉPINE

Université de Sherbrooke, Collectif CLÉ, QC J1K 2R1 Sherbrooke, Canada 